\title{
Charcot neuroarthropathy of the knee due to idiopathic sensory peripheral neuropathy
}

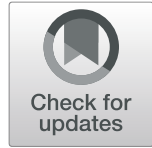

Qian-Hao Yang ${ }^{1}$, Peichun Hsu', You-Shui Gao ${ }^{12^{*}}$ (D) and Chang-Qing Zhang ${ }^{1}$

\begin{abstract}
Background: Charcot neuroarthropathy is a systemic disease that generates pathological changes in the musculoskeletal system, causing instability, dislocations, and deformities. Charcot neuroarthropathy of the knee, due to either diabetes mellitus or syringomyelia, is anecdotally reported with the epidemic of the diseases. However, idiopathic sensory peripheral neuropathy can inflict osteoarticular structures directly, inducing a dysfunctional Charcot neuroarthropathy. An early diagnosis and effective relief of the symptomatic deformity is essential for the treatment.
\end{abstract}

Case presentation: We report the case of a patient with idiopathic sensory peripheral neuropathy who presented with a swelling right knee, as well as distorted and painless gait disorder, diagnosed as Charcot neuroarthropathy of the knee. Partial weight bearing with a hinged knee brace was used to correct the abnormal alignment and gait posture, and bisphosphonates were prescribed to decrease pathological bone resorption. Although the alignment and Knee Society Score got a gradual deterioration, the combination of orthosis and pharmacy could alleviate the symptom to a certain extent.

Conclusion: The diagnosis of Charcot neuroarthropathy of the knee is rare that requiring early diagnosis. The presence of features, including painlessness, numbness, and deformed arthropathy following chronic-

onset algesthesia loss should be taken carefully.

Keywords: Charcot arthropathy, Idiopathic sensory peripheral neuropathy, Knee

\section{Background}

Charcot neuroarthropathy is a systemic disease that generates pathological changes in the musculoskeletal system, causing instability, dislocations, and deformities [1], first named by Jean Martin Charcot (1829-1893) in 1868 [2]. Diabetes mellitus and neurosyphilis are the most common causes of Charcot neuroarthropathy these days, mostly affecting the foot and ankle [3].

Charcot neuroarthropathy is reported to be closely associated with diabetes mellitus [4], syringomyelia [5], neurosyphilis [2], and idiopathic neuropathy [6], which might be misdiagnosed in many cases. With an increasing incidence of arthropathy, there is controversy about the treatment of Charcot neuroarthropathy in the knee. Total knee arthroplasty (TKA), arthrodesis, and conservative treatment

\footnotetext{
* Correspondence: gaoyoushui@sjtu.edu.cn

${ }^{1}$ Department of Orthopedic Surgery, Shanghai Jiao Tong University Affiliated Sixth People's Hospital, Shanghai, China

${ }^{2}$ Centre for Orthopaedic Research, Faculty of Health and Medical Sciences,

The University of Western Australia, Nedlands, WA 6009, Australia
}

should be considered appropriately in Charcot neuroarthropathy of diverse causes and stages. In the scenario of concomitant osteomyelitis, amputation might become the treatment of choice [7]. Herein, we present a case of Charcot neuroarthropathy of the knee following idiopathic sensory peripheral neuropathy.

\section{Case presentation}

A 40-year-old man presented to the orthopedic clinic with a 2-week history of swelling in the right knee and a 1-year history of painless gait disorder. He had previously been diagnosed with idiopathic sensory peripheral neuropathy, for which he had multiple fingertips falling off in his early teens and left leg amputation 5 years prior following massive painless ulceration (Fig. 1). The diagnosis of idiopathic sensory peripheral neuropathy follows the approach to the evaluation of peripheral neuropathies [8], evidenced as the unique clinical manifestation of distally predominant and symmetrical sensory abnormalities, and electrophysiological findings of mainly 


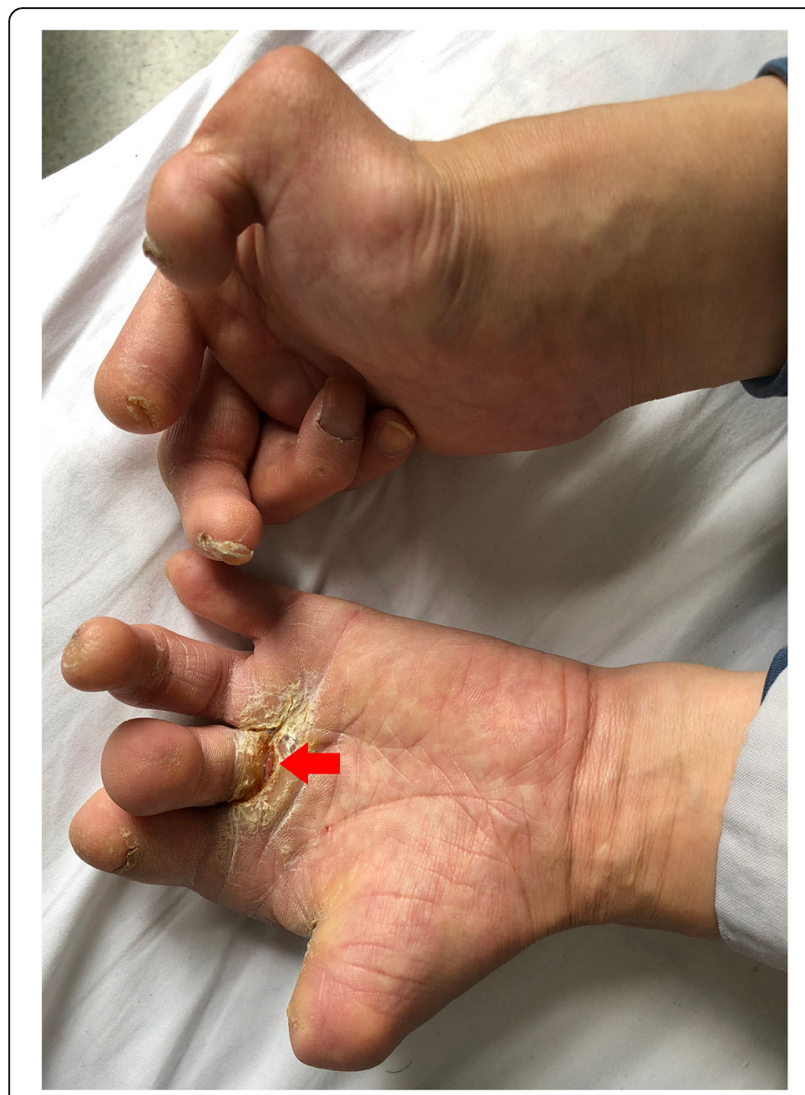

Fig. 1 Multiple digital tips fall off naturally, in concomitant with uncontrollable and painless ulceration. The remnant fingers are deformed, with obvious palmar ulcer as indicated

axonal damage. Charcot-Marie-Tooth disease was excluded due to his normal motor function. There was no history of diabetes mellitus and neurosyphilis. He also had no family history of inherited diseases.

On physical examination, a valgus distortion of the knee was noted, with significantly restricted ranges of flexion $\left(105^{\circ}\right)$ and extension $\left(10^{\circ}\right)$. Inspired by a recent study [9], we used the system of Knee Society Score (KSS) to evaluate the function. The knee score was 46 and the function score was 40 on admission. There was a slight deep tenderness over the superolateral aspect of the patella and the lateral femoral condyle. Despite gross deformity and distinct instability, he complained of no pain during daily activities and sleep. Paracentesis yielded bloody effusions, both negative for biochemical examinations and microbial culture. Laboratory studies showed slight elevation in alkaline phosphatase (ALP) levels (147 U/L; reference: 34-104 U/L) at admission, reflecting abnormal absorption of bone metabolism. Infection was excluded due to the normal ranges of leukocyte count $\left(4.7 \times 10^{9} / \mathrm{L}\right.$; reference: $3.5 \times 10^{9}-9.5 \times$ $\left.10^{9} / \mathrm{L}\right)$, C-reactive protein $(10.36 \mathrm{mg} / \mathrm{L}$; reference: $0.00-$ $10.00 \mathrm{mg} / \mathrm{L})$, and erythrocyte sedimentation rate (12 $\mathrm{mm} / \mathrm{h}$; reference: $0-21 \mathrm{~mm} / \mathrm{h}$ ) along with negative microbiological and cytological examination of the repeated fluid aspiration. The coagulation and syphilis tests were normal without evidence of hemophilic arthritis and syphilis-induced Charcot arthropathy. Plain radiographs and CT scans showed apparent widening of the joint space and multiple abrasions of the lateral femoral condyle, with abnormal debris close to the posterior plateau (Figs. 2, 3). Stress radiography was not conducted due to the high risk of pathologic fractures and incompetency of pain complaint. Plain radiographs, captured under supine and neutral position, showed the femorotibial angle (FTA) was $165^{\circ}$. Magnetic resonance imaging revealed a wide impairment of the anterior cruciate ligament, lateral collateral ligament, and meniscus lateralis with massive joint effusions (Fig. 4). Idiopathic sensory peripheral neuropathy-induced Charcot neuroarthropathy was diagnosed in collaboration with neurologists.

Partial weight bearing with a hinged knee brace was advocated to correct the abnormal alignment and gait posture, followed by a gradual increase in flexion and extension over a 3-month period for clinical improvement. However, the significant disability determined the joint motion should be protected by the orthosis. Mild swelling of the knee was found in the lateral compartment, but there was no tenderness or redness. Additionally, he was administered physical therapy and rehabilitation along with bisphosphonates to decrease pathological bone resorption. ALP was dynamically monitored and found to be maintained between 108 134 U/L (reference: $50-136 \mathrm{U} / \mathrm{L})$. Plain radiographs at the 12-month follow-up showed the valgus knee with the FTA of $159^{\circ}$, with a slight deterioration restricted in the lateral compartment (Fig. 5). The knee was reevaluated with KSS, showing the knee score dropped to 41 and the function score maintained 40 . The range of motion was $10^{\circ} \sim$ $105^{\circ}$, reflecting the flexion and extension in the sagittal plane was not affected. Considering the potential risks of arthroplasty and inherent disadvantages of arthrodesis, protected weightbearing with the orthosis was recommended as the current treatment of choice [10].

\section{Discussion and conclusions}

Historically, the 2 dominant pathophysiological causes of Charcot neuroarthropathy included the neurotraumatic (abnormal sensory innervation, repeated microtrauma, and unregulated inflammatory cascade) and neurovascular theories (hypervascular region in subchondral bone, vascular shunting, and subsequent osteopenia) [1, 3]. Peripheral neuropathy caused by diabetes mellitus is the main cause of Charcot neuroarthropathy of the knee. While the idiopathic sensory peripheral neuropathy in our case can cause chronic-onset loss of algesthesia, inducing hypalgesia, numbness, and deformed arthropathy. 


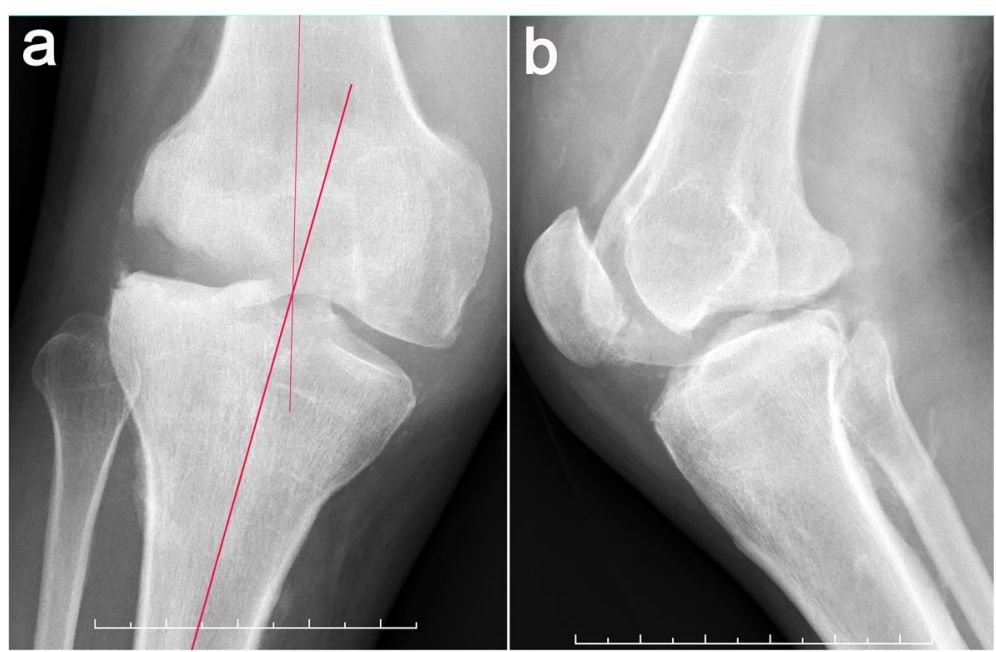

Fig. 2 Anteroposterior (a) and lateral (b) plain radiographs of the right knee show a valgus knee, with a destructive femoral condyle. The knee is swollen, distorted, and painless, with a femorotibial angle of $165^{\circ}$

The primary degeneration of the dorsal root ganglion and trigeminal ganglion sensory neurons can cause gait ataxia, proprioceptive sensory loss, unnoticed damage, as well as repetitive microtrauma to the weight-bearing joint, contributing to Charcot neuroarthropathy $[11,12]$. If not diagnosed and intervened promptly, it may result in severe complications, such as fractures and joint dislocations.

Charcot neuroarthropathy can occur secondary to syringomyelia, syphilis, diabetic neuropathy, alcoholic peripheral neuropathy, and idiopathic neuropathy. Diabetes is the most common etiology. Charcot neuroarthropathy is considered a major risk factor for below-the-knee amputation among the diabetics [1], while upper extremity can also be affected as a result of syringomyelia [5]. The diagnosis of Charcot neuroarthropathy is based primarily on thorough history and physical examination, including features, such as loss of protective sensation, presence of ulceration, and warm, swollen, erythematous foot and/or ankle. A combination of autonomic neuropathy, motor neuropathy, and sensory neuropathy results in osteopenia, joint instability, and increased loads on joints. The signs and symptoms of idiopathic sensory peripheral neuropathy-induced Charcot neuroarthropathy are consistent with non-length dependent nerve-fiber degeneration. The loss of sensory neurons in the dorsal root ganglia induce degeneration of short and long peripheral axons and central sensory projections in the posterior columns. Idiopathic sensory peripheral neuropathy induced Charcot neuroarthropathy, unlike others, is a rare disorder with unique characteristics, including deformities of fingertips and asymmetrical sensory disturbances.

Neuropathic arthropathy can be classified into four stages using standard radiology (prodromal, developmental,

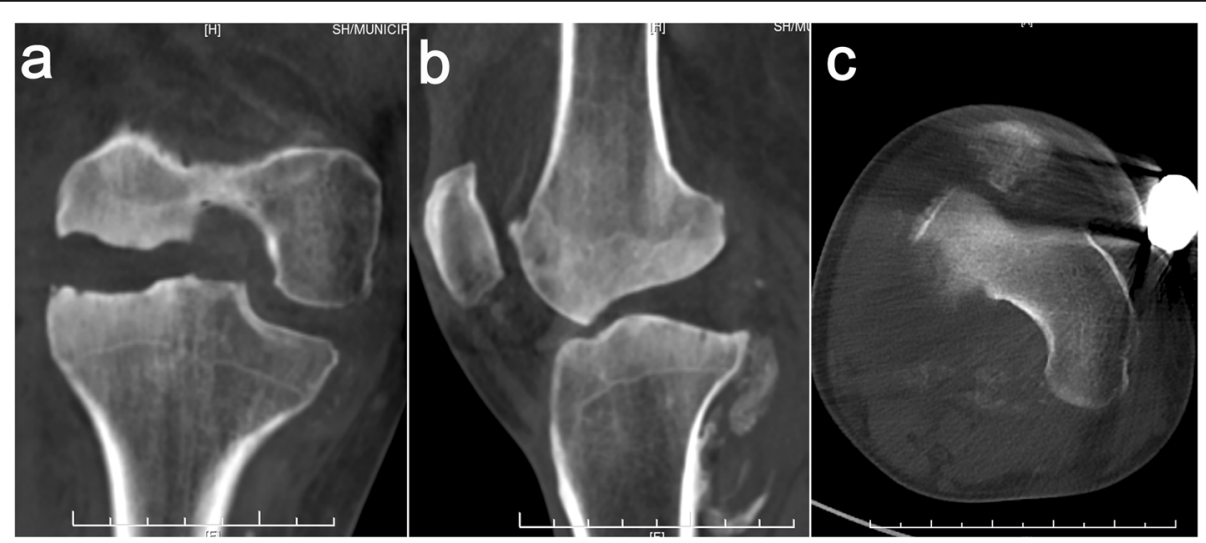

Fig. 3 CT scanning of the right knee shows apparent widening of the joint space due to increased effusions (a) and aberrant morphology of lateral femoral condyle (b), with abnormal debris close to the posterior plateau (b, c) 


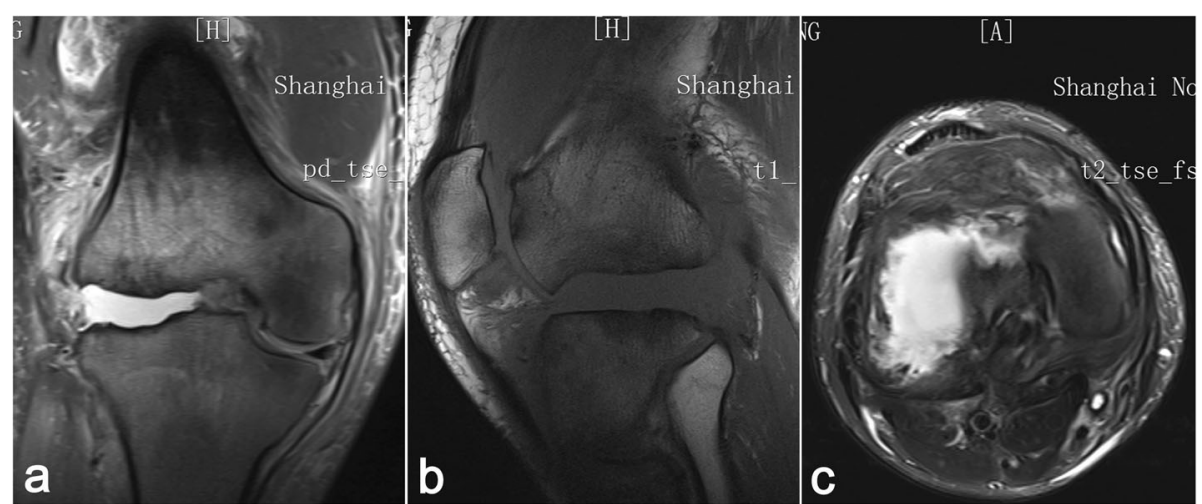

Fig. 4 Magnetic resonance imaging reveals a wide disruption of anterior cruciate ligament, lateral collateral ligament and meniscus lateralis (a, b), with significantly increased effusions. The lateral femoral condyle is almost entirely destructed while the medial condyle is uninvolved (c)

coalescence, and reconstructive) [10]. Identification of Charcot neuroarthropathy of the knee in Stage 0 and I have important therapeutic implications. The immobilization and non-weight bearing of the knee may prevent progression of skeletal destruction and deformity. Antiresorptive therapy, especially with bisphosphonates, has been revealed to have a modest effect on Charcot neuroarthropathy [13], inhibiting excessive osteoclast activation and proinflammatory cytokine response [14]. Total knee arthroplasty (TKA) and arthrodesis should be employed only in the reconstruction or coalescence stages [15], similar to a salvage operative treatment. Although TKA is satisfactory for most patients [16], the complications due to fragile bone and ligamentous laxity still exists [17], including periprosthetic fracture, aseptic loosening, instability, and infection [18]. Knee arthrodesis is accompanied by major functional limitations, resulting in the restriction of knee motion. Bisphosphonates were prescribed due to the nature of progressive destruction of Charcot neuroarthropathy. Bisphosphonates were beneficial to control the level of ALP, which was monitored through the observation. However, with a brief literature review, we found most of previous studies did not detect any biomarkers associated with bone absorption [5, 6, 9, 19-22]. Although Charcot neuroarthropathy is not a bone metabolism disorder in nature, a dynamic monitor of serum biomarkers can provide substantial reference to modulate bone homeostasis and alleviate skeletal destruction. The activity of osteoclast and underlying inflammation should be evidenced by essential mechanistic investigations in the future [1].

To the best of our knowledge, this is the first case of Charcot neuroarthropathy due to idiopathic sensory peripheral neuropathy. The patient had an impressive history of multiple fingertips abscission and left leg amputation following painless ulceration. Nerve conduction studies confirmed the presence of idiopathic sensory peripheral neuropathy. The swelling of the right knee and painless gait disorder were the primary clinical features, consistent

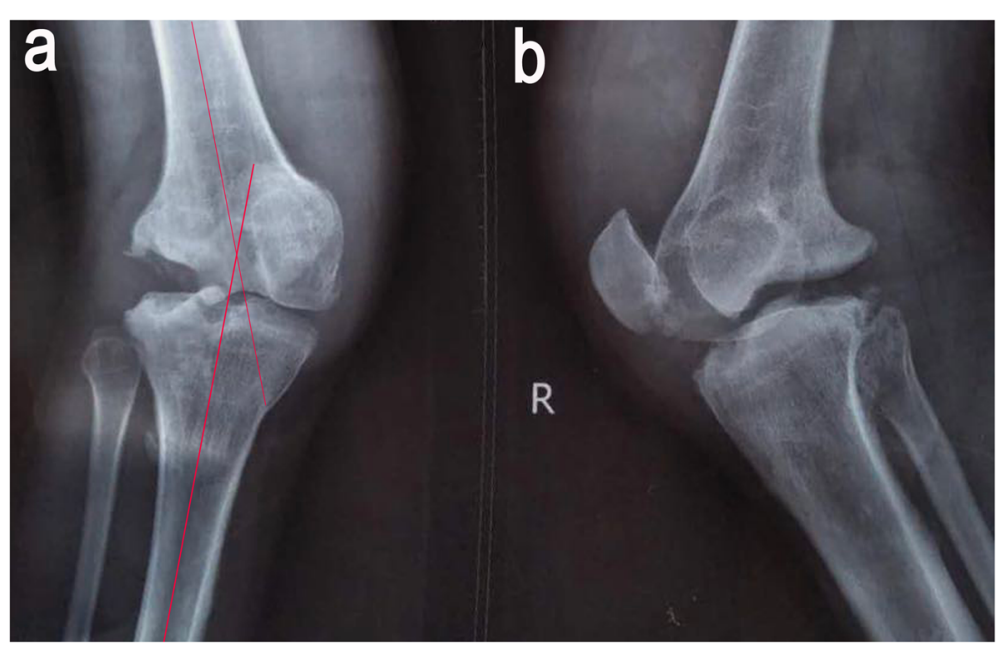

Fig. 5 Anteroposterior (a) and lateral (b) plain radiographs of the knee at the 12-month follow-up showed the lesion was restricted in the lateral compartment. The femorotibial angle has progressed to $159^{\circ}$ 
with the diagnosis of neuropathic arthropathy of the knee. Moreover, only the lateral compartment of the knee was significantly involved, with an obvious valgus deformity, detected promptly before total joint destruction. Our case had satisfactory clinical outcomes from periods of nonoperative management in Stage I.

In summary, the diagnosis of Charcot neuroarthropathy of the knee is rare and is considered as the accumulated effect of peripheral neuropathy. Although an early diagnosis of Charcot arthropathy cannot alter natural course of the disease, it is beneficial to relieve symptoms and prevent severe complications. The presence of features, including painlessness, numbness, and deformed arthropathy following chronic-onset algesthesia loss increases the likelihood of idiopathic sensory peripheral neuropathy-induced Charcot neuroarthropathy.

\section{Abbreviations}

ALP: Alkaline phosphatase; FTA: Femorotibial angle; KSS: Knee Society Score; TKA: Total knee arthroplasty

\section{Acknowledgements}

We thank the patient for agreeing to publication of his case. Additionally, we sincerely thank Prof. You-Fen Tan from Jiangsu Provence Hospital of TCM, Prof. Yu-Pu Guo from Peking Union Hospital and Prof. Fan Liu from Nantong University Affiliated Hospital, for their contributions in the diagnosis and care of the patient. Although the disease is depressing, our patient is still filled with enthusiasm for life.

\section{Authors' contributions}

Q-HY and PH collected and analyzed the clinical data of the patient. Y-SG, and C-QZ undertook patient rehabilitation. All authors cared for the patient and approved the final manuscript.

\section{Funding}

No funding was obtained for this study.

\section{Availability of data and materials}

All data generated or analyzed during this study are included in this published article.

\section{Ethics approval and consent to participate}

All procedures were part of the standard medical care, and the need for ethics approval and consent to participate was waived.

\section{Consent for publication}

Written informed consent was obtained from the patient for publication of this case report and any accompanying images. The proof of consent to publish from study participants can be requested at any time.

\section{Competing interests}

The authors declare that they have no competing interests.

Received: 8 May 2019 Accepted: 9 October 2019

Published online: 30 October 2019

\section{References}

1. Dodd A, Daniels TR. Charcot neuroarthropathy of the foot and ankle. J Bone Joint Surg Am. 2018;100(8):696-711.

2. Wukich DK, Sung W. Charcot arthropathy of the foot and ankle: modern concepts and management review. J Diabetes Complicat. 2009;23(6):409-26.

3. Kucera T, Urban K, Sponer P. Charcot arthropathy of the knee. A case-based review. Clin Rheumatol. 2011:30(3):425-8.

4. Patel A, Saini AK, Edmonds ME, Kavarthapu V. Diabetic neuropathic arthropathy of the knee: two case reports and a review of the literature. Case Rep Orthop. 2018;2018:9301496.
5. Li G, Ding Y, Zhang C, Huang H. Syringomyelia with left knee Charcot arthropathy: a case report. Br J Neurosurg. 2018. p. 1-2.

6. Hanson LF, Hanson CG, Barner KL. Idiopathic Charcot arthropathy of the knee presenting as a Bicondylar Tibial plateau fracture. J Am Acad Orthop Surg Glob Res Rev. 2018;2(7):e032.

7. Berli M, Vlachopoulos L, Leupi S, Boni T, Baltin C. Treatment of Charcot neuroarthropathy and osteomyelitis of the same foot: a retrospective cohort study. BMC Musculoskelet Disord. 2017;18(1):460.

8. England JD, Asbury AK. Peripheral neuropathy. Lancet. 2004;363(9427): 2151-61.

9. Tibbo ME, Chalmers BP, Berry DJ, Pagnano MW, Lewallen DG, Abdel MP. Primary total knee arthroplasty in patients with neuropathic (Charcot) arthropathy: contemporary results. J Arthroplast. 2018;33(9):2815-20.

10. Rosenbaum AJ, DiPreta JA. Classifications in brief: Eichenholtz classification of Charcot arthropathy. Clin Orthop Relat Res. 2015:473(3):1168-71.

11. Sghirlanzoni A, Pareyson D, Lauria G. Sensory neuron diseases. Lancet Neurol. 2005:4(6):349-61.

12. Callaghan BC, Price RS, Chen KS, Feldman EL. The importance of rare subtypes in diagnosis and treatment of peripheral neuropathy: a review. JAMA Neurol. 2015;72(12):1510-8.

13. Pitocco D, Ruotolo V, Caputo S, Mancini L, Collina CM, Manto A, et al. Sixmonth treatment with alendronate in acute Charcot neuroarthropathy: a randomized controlled trial. Diabetes Care. 2005;28(5):1214-5.

14. Jostel A, Jude EB. Medical treatment of Charcot neuroosteoarthropathy. Clin Podiatr Med Surg. 2008;25(1):63-9 vi-vii.

15. Zeng M, Xie J, Hu Y. Total knee arthroplasty in patients with Charcot joints. Knee Surg Sports Traumatol Arthrosc. 2016;24(8):2672-7.

16. Hayashi S, Okada T, Horita K. Bilateral TKA for Charcot arthropathy associated with multiple levels of spinal canal stenoses: a case report and review of literature. J Orthop. 2017;14(1):77-80.

17. Kim YH, Kim JS, Oh SW. Total knee arthroplasty in neuropathic arthropathy. J Bone Joint Surg Br. 2002;84(2):216-9.

18. Parvizi J, Marrs J, Morrey BF. Total knee arthroplasty for neuropathic (Charcot) joints. Clin Orthop Relat Res. 2003;416:145-50.

19. Chalmers BP, Tibbo ME, Trousdale RT, Lewallen DG, Berry DJ, Abdel MP. Primary total hip arthroplasty for Charcot arthropathy is associated with high complications but improved clinical outcomes. J Arthroplast. 2018; 33(9):2912-8.

20. Figueiredo A, Ferreira R, Alegre C, Fonseca F. Charcot osteoarthropathy of the knee secondary to neurosyphilis: a rare condition managed by a challenging arthrodesis. BMJ Case Rep. 2018;2018:bcr-2018.

21. Nambiar M, Onggo JR, Pai V. Neuropathic arthropathy of the shoulder joint secondary to a syringomyelia. BMJ Case Rep. 2018;11(1):e228228.

22. Choudhury P, Mohapatra A, Kharat A, Chauhan V. Neuropathic arthropathy of the shoulder joint caused by syringomyelia: report of a rare case. BMJ Case Rep. 2019;12(3):e229494.

\section{Publisher's Note}

Springer Nature remains neutral with regard to jurisdictional claims in published maps and institutional affiliations.

Ready to submit your research? Choose BMC and benefit from:

- fast, convenient online submission

- thorough peer review by experienced researchers in your field

- rapid publication on acceptance

- support for research data, including large and complex data types

- gold Open Access which fosters wider collaboration and increased citations

- maximum visibility for your research: over $100 \mathrm{M}$ website views per year

At $\mathrm{BMC}$, research is always in progress.

Learn more biomedcentral.com/submissions 